\title{
Prognostic Significance of Skeletal Muscle Loss During Early Postoperative Period in Elderly Patients with Esophageal Cancer
}

\author{
Mary J. Fidler, MD', Jeffrey A. Borgia, $\mathrm{PhD}^{2}$, Philip D. Bonomi, $\mathrm{MD}^{3}$, and Palmi Shah, $\mathrm{MD}^{4}$ \\ ${ }^{1}$ Medical Oncology, Rush University Medical Center, Chicago; ${ }^{2}$ Pathology, Rush University Medical Center, Chicago; \\ ${ }^{3}$ Medical Oncology, Rush University Medical Center, Chicago; ${ }^{4}$ Radiology, Rush University Medical Center, Chicago
}

In this issue of Annals of Surgical Oncology, Takahashi et al. ${ }^{1}$ report that skeletal muscle reduction in the highest tertile of their patient series was associated with worse cancer-free and overall survival after an oncologic esophagectomy. The patients were 65 years of age or older, had no cancer recurrence at 4 months, and had undergone $\mathrm{R} 0$ resections.

The skeletal muscle index (SMI) analysis remained significant in the multivariate analysis for recurrence-free and overall survival. In fact, SMI loss in the highest tertile of patients had a hazard ratio (HR) of 5.405 for death ( $p<0.001 ; 95 \%$ confidence interval [CI], 3.514-8.314) and showed similar findings for recurrence-free survival. The patients with the highest tertile of skeletal muscle loss had more node-positive pathology at the time of resection, suggesting that the presence of microscopic disease may have correlated with the loss of skeletal muscle.

Pre-therapeutic sarcopenia in esophageal cancers is highly prevalent as well as significantly and independently associated with postoperative complications, chemotherapy-induced toxicity, and poor survival in cancer patients, as shown in a recent systematic review. ${ }^{2}$ Although we have no information on the use of postoperative enteral feeding and skeletal muscle index in this setting, enteral feeding has not demonstrated improvement in terms of postoperative weight loss. ${ }^{3}$

\footnotetext{
(C) Society of Surgical Oncology 2019
}

First Received: 25 July 2019;

Published Online: 30 August 2019

M. J. Fidler, MD

e-mail: Mary_Fidler@rush.edu
Nagata et al. ${ }^{4}$ also studied postoperative skeletal muscle mass after esophagectomy and found that reduced skeletal muscle in the psoas muscle 6 months after esophagectomy did not correlate with preoperative nutrition status or neoadjuvant therapy. Their series showed no association with cancer recurrence, although the patients with presarcopenia at the 6-month mark did have more pneumonia and a doubling of 12 -month mortality $(24.1 \%$ vs $11.7 \%$; $p=0.11$.

The most accepted and current definition of sarcopenia as per the European Working Group on Sarcopenia in Older People (EWGSOP) is the accelerated loss of skeletal muscle mass and function. ${ }^{5}$ Sarcopenia is a hallmark of cancer cachexia, and its diagnosis is heavily reliant on measurement of the muscle mass. ${ }^{6}$ Among the objective noninvasive methods for evaluating sarcopenia, computed tomography (CT) and magnetic resonance imaging (MRI) are considered gold standards. ${ }^{7}$

Quantification and quality of muscle mass can easily be derived from CT and positron emission tomography (PET)$\mathrm{CT}$ studies routinely performed for staging and restaging in oncology patients without any extra cost. In particular, CTderived muscle mass at L3 has been shown to correlate well with whole-body muscle mass. ${ }^{8}$ Although semiautomatic muscle segmentation is the current trend for obtaining the CT-derived measures of muscle mass and myostatosis, the variations in the $\mathrm{CT}$ acquisition parameters and the image analysis techniques make it difficult to compare data across techniques. ${ }^{9}$

Although successful therapeutic targeting of cancer cachexia may not eliminate microscopic disease, it could alleviate some of the postoperative complications associated with cachexia. Findings have demonstrated that patients after esophagectomy have decreased ghrelin secretion, which can lead to early satiety, decreased 
appetite, less growth hormone secretion, and inflammation. ${ }^{10}$ Using ghrelin agonists after esophagectomy for select patients could be a strategy to mitigate sarcopenia. This strategy led to an increase in skeletal muscle mass in a placebo-controlled randomized trial with stage 4 lung cancer patients, although in the post-esophagectomy setting, correlations with weight gain have varied. ${ }^{11-14}$

Additional cachexia-reversing strategies include targeting chronic inflammation. Muscle formation is tightly linked to immune modulation. Pro-cachectic cytokines, including growth differentiation factor 15 (GDF-15), interleukin-1 (IL-1), IL-6, tumor necrosis factor-alpha (TNF- $\alpha$ ), and interferon-gamma (INF- $\gamma$ ), released by either the tumor or immune cells, activate proteolysis, myocyte apoptosis, or decreased amino acid transport and regeneration. ${ }^{15}$ Strategies to reverse cachexia by reducing inflammation with nonsteroidal antiinflammatory agents (NSAIDS) have been deemed not useful, although one randomized controlled trial is ongoing, applying ibuprofen with nutritional support and exercise in the intervention group. ${ }^{16,17}$ The hypothesis is that cachexia intervention may be more successful during the early stages.

Chronic inflammation is associated with decreased effectiveness of cytotoxic $\mathrm{T}$ cells, which are the hypothesized means of killing cancer cells with the use of programmed death-1 (PD-1) or programmed death ligand-1 (PD-L1) inhibitors. ${ }^{18}$ Patients with low postoperative sarcopenia could be candidates for immune checkpoint inhibitors, especially because sarcopenia may be a marker for microscopic disease.

The addition of a new International Classification of Diseases (ICD)-10 code for sarcopenia points to recognition of this as a distinct disease entity ${ }^{19}$ while encouraging additional research in diagnosis and treatment of this disease. Evolving consensus definitions and future availability of fully automated algorithms for segmenting CT images to quantify body composition based on a deep learning approach will allow for the effective integration of CTbased diagnosis of sarcopenia into patient care. ${ }^{20}$

\section{REFERENCES}

1. Takahashi K, Wantanabe M, Kuzuki R, et al. Prognostic significance of skeletal muscle loss during early postoperative period in elderly patients with esophageal cancer. Ann Surg Oncol. 2019. https://doi.org/10.1245/s10434-019-07616-0.

2. Pamoukdjian F, Bouillet T, Levy V, Soussan M, Zelek L, Paillaud E. Prevalence and predictive value of pre-therapeutic sarcopenia in cancer patients: a systematic review. Clin Nutr. 2018;37:1101-13.

3. Froghi F, Sanders G, Berrisford R, et al. A randomised trial of post-discharge enteral feeding following surgical resection of an upper gastrointestinal malignancy. Clin Nutr. 2017;36:1516-9.
4. Nagata $K$, Tsujimoto $H$, Nagata $H$, et al. Impact of reduced skeletal muscle volume on clinical outcome after esophagectomy for esophageal cancer: a retrospective study. Medicine. 2018;97:e11450.

5. Cruz-Jentoft AJ, Bahat G, Bauer J, et al. Sarcopenia: revised European consensus on definition and diagnosis. Age Ageing. 2019;48:16-31.

6. Fearon K, Strasser F, Anker SD, et al. Definition and classification of cancer cachexia: an international consensus. Lancet Oncol. 2011;12:489-95.

7. Beaudart C, McCloskey E, Bruyere O, et al. Sarcopenia in daily practice: assessment and management. BMC Geriatr. 2016;16:170.

8. Mourtzakis M, Prado CM, Lieffers JR, Reiman T, McCargar LJ, Baracos VE. A practical and precise approach to quantification of body composition in cancer patients using computed tomography images acquired during routine care. Appl Physiol Nutrition Metab. 2008;33:997-1006.

9. Amini B, Boyle SP, Boutin RD, Lenchik L. Approaches to assessment of muscle mass and myosteatosis on computed tomography (CT): a systematic review. J Gerontol Ser A Biol Sci Med Sci. 2019. https://doi.org/10.1093/gerona/glz034.

10. Nakazato M, Murakami N, Date Y, et al. A role for ghrelin in the central regulation of feeding. Nature. 2001;409:194-8.

11. le Roux CW, Neary NM, Halsey TJ, et al. Ghrelin does not stimulate food intake in patients with surgical procedures involving vagotomy. J Clin Endocrinol Metab. 2005;90:4521-4.

12. Murphy CF, le Roux CW (2016) The neurobiological impact of ghrelin suppression after oesophagectomy. Int J Mol Sci. 18:E35.

13. Temel JS, Abernethy AP, Currow DC, et al. Anamorelin in patients with non-small cell lung cancer and cachexia (ROMANA 1 and ROMANA 2): results from two randomised, double-blind, phase 3 trials. Lancet Oncol. 2016;17:519-31.

14. Yamamoto K, Takiguchi S, Miyata H, et al. Randomized phase II study of clinical effects of ghrelin after esophagectomy with gastric tube reconstruction. Surgery. 2010;148:31-8.

15. Lerner L, Hayes TG, Tao N, et al. Plasma growth differentiation factor 15 is associated with weight loss and mortality in cancer patients. J Cachexia Sarcopenia Muscle. 2015;6:317-24.

16. Shinko D, Diakos CI, Clarke SJ, Charles KA. Cancer-related systemic inflammation: the challenges and therapeutic opportunities for personalized medicine. Clin Pharmacol Ther. 2017;102:599-610.

17. Solheim TS, Laird BJA, Balstad TR, et al. Cancer cachexia: rationale for the MENAC (multimodal-exercise, nutrition, and antiinflammatory medication for cachexia) trial. BMJ Support Palliat Care. 2018;8:258-65.

18. Zhao Q, Yu J, Meng X. A good start of immunotherapy in esophageal cancer. Cancer Med. 2019. https://doi.org/10.1002/cam4. 2336.

19. Anker SD, Morley JE, von Haehling S. Welcome to the ICD-10 code for sarcopenia. $J$ Cachexia Sarcopenia Muscle. 2016;7:512-4.

20. Weston AD, Korfiatis P, Kline TL, et al. Automated abdominal segmentation of CT scans for body composition analysis using deep learning. Radiology. 2019;290:669-79.

Publisher's Note Springer Nature remains neutral with regard to jurisdictional claims in published maps and institutional affiliations. 